\title{
Importancia de los mecanismos de participación ciudadana en los sistemas democráticos
}

Importance of the mechanisms for citizen participation in the democratic systems

\author{
Joan López C. ${ }^{1}$ \\ (iD https://orcid.org/0000-0002-4656-4691 \\ Universidad Politécnica de Nicaragua, UPOLI
}

\section{Como referencias este artículo:}

López C., J. (2020). Importancia de los mecanismos de participación ciudadana en los sistemas democráticos. Revista Ciencia Jurídica y Política, 31-44. Recuperado de: https://portalderevistas.upoli.edu.ni/index.php/5-revciencasjuridicasypoliticas/article/view/634

\section{RESUMEN}

En este trabajo, se señala la importancia que conlleva en un sistema democrático el que existan mecanismos formales de participación ciudadana como una forma de relación socio-estatal. La ciudadanía asocia su participación con la materialización de ejercer su derecho al voto para elegir al mandatario de su nación, más allá de eso no se percata que el involucramiento de su participación en la agenda local podría obtener un mayor alcance para combatir la carencia de credibilidad y legitimidad que existe en el mismo sistema de gobierno. Si bien es cierto existen diversos mecanismos de participación ciudadana también es cierto que la ciudadanía los desconoce, no sabe cómo utilizarlos o bien no los encuentra interesantes. Por el otro lado, no hay una estimulación activa en las iniciativas gubernamentales para que se logre un cambio en la percepción de los ciudadanos con estos mecanismos; por lo que se debe de estudiar de manera reflexiva a que se enfrentan los ciudadanos para hacer uso de su derecho de participación por medio de los mecanismos formales de participación ciudadana y la envergadura que tiene en los sistemas democráticos.

Palabras clave: Ciudadanía, Gobierno local, Mecanismos de Participación Ciudadana, Sistemas democráticos.

\begin{abstract}
In this work, it points out the importance of formal mechanisms for citizen participation as a form of socio- state relationship in a democratic system is noted. Citizenship associates its participation with the materialization of the opportunity to vote to elect the president of its nation, beyond that it is not realize the involvement of the participation in the local agenda could obtain a greater scope to combat the lack of credibility and legitimacy that exists in the system of government itself. Although it is true that they exist diverse mechanisms, and it is also true that the citizenship does not know how to use them or does not find them interesting. On the other hand, there is not an active stimulation in government initiatives to bring a change in the perception of the citizens through these mechanisms; therefore, it is necessary to study in a reflective way to what the citizens face to make use of their rights through the formal mechanisms of citizen participation and the extent that is has in the democratic systems.
\end{abstract}

Key word: Citizenship, Local government, Citizen Participation Mechanisms, Democratic systems.

\footnotetext{
${ }^{1}$ Candidata a Máster en Ciencias Políticas por la Facultad de Ciencias Políticas y Relaciones Internacionales de la UANL. Licenciatura en Derecho por la Universidad Politécnica de Nicaragua UPOLI. Licenciatura en Administración Turística y Hotelera UPOLI. Cuenta con Diplomados en Derecho de Familia por la Universidad Politécnica de Nicaragua; Docente Suplente en actividades de Becaria de CONACYT en la Facultad de Ciencias Políticas y Relaciones Internacionales de la UANL. Secretaria Judicial de la Corte Suprema de Justicia Nicaragua.
} 


\section{INTRODUCCIÓN}

Se han hecho múltiples investigaciones acerca de la participación ciudadana y la importancia de los mecanismos formales como un papel legitimante para la administración de un gobierno. Esta investigación se encamina en el contexto de que los ciudadanos no solo pueden participar en la democracia de un país mediante el voto, sino que existen diversos métodos establecidos en las legislaciones de los países como un cuerpo normativo donde se detallan los mecanismos de participación ciudadana que los mismos actores pueden utilizar para tener un mayor involucramiento en las decisiones de la administración pública. El objetivo es conocer que tanto interés existe entre de la administración pública y los ciudadanos en cuanto a la participación ciudadana.

La investigación inicia con el interés de una concepción de la participación ciudadana como una evolución de los estados democráticos. Brugué (2004) expresa que esto no es más que la necesidad de un involucramiento de los ciudadanos en la agenda local para combatir los déficits de credibilidad y legitimidad a los que están expuestos los gobiernos.

Otro aspecto que da relevancia a este estudio es la conexión que debe existir entre estado y ciudadanía como lo establece Bourgon (2010) donde manifiesta que es importante que los actores participativos tengan una estimulación activa, así como un conocimiento de los mecanismos formales que se pueden utilizar y lo trascendental que es para los gobiernos locales mantener un canal de comunicación entre ellos y los ciudadanos.

Es por ello por lo que el valor de la comunicación permite el apoyo a las iniciativas gubernamentales, generando consigo una nueva forma de asumir los asuntos públicos (Bourgon, 2010). Lograr el cambio de percepción que tienen los ciudadanos puede significar grandes desafíos para la administración local ya que la estrategia es abrir espacios de participación a través de los mecanismos formales, pero también que los ciudadanos tengan conocimiento de la existencia de estos mecanismos y la forma en cómo pueden accionarlos. (Pastor y García, 2002). 


\section{ANTECEDENTES HISTÓRICOS DE LA PARTICIPACIÓN CIUDADANA}

Es importante situar el contexto de la participación ciudadana, por lo que hay que tomar en cuenta el nacimiento de esta figura, es por ello que es importante distinguir la diferencia entre ciudadanía y democracia dentro del pensamiento político.

Si bien es cierto la democracia es un sistema político que defiende la soberanía del pueblo y el derecho del mismo a elegir y controlar a sus gobernantes, la ciudadanía es un conjunto formado por los ciudadanos de una nación o pueblo, conceptos que a simple vista están vinculados pero el pensamiento político demuestra que si no existe democracia no puede existir ciudadanía. (Bautista, 2015).

Aunque el concepto de ciudadanía se relaciona con el ámbito de la modernidad, su nacimiento según Horrach (2009) fue hace unos 2,500 años en la época de la Grecia clásica, afectando poco a poco las esferas de la realidad.

Del mismo modo en Grecia dieron inicio grandes acontecimientos, entre ellos están la democracia y la filosofía. Las características básicas se dan dentro del desarrollo de la idea del demos (pueblo) y la participación ciudadana, consecuentemente del sujeto político donde la actividad directa de los ciudadanos fue de una posición de control a un ejercicio directo del poder. (Arteta, 2008).

Al paso del tiempo se encuentra en absoluto que el tema de la ciudadanía haya tenido una importancia tan decisiva. La ciudadanía estructuraba fuertemente de una o de otra manera la vida cotidiana.

De igual forma la caída del Imperio Romano acabó en la práctica con la ciudadanía, pues Horrach manifiesta: "La autocracia bizantina no le dio margen de maniobra; también, los pueblos bárbaros que conquistaron Europa se romanizaron progresivamente y adoptaron la fe cristiana. Al abandono de la ciudadanía corresponde el olvido de la idea de democracia, que, tras el experimento griego, es sustituida por otros modelos políticos menos igualitarios” (Horrach, 2009, pág. 9). 
A pesar de ello, la idea esencial de ciudadanía nunca pudo ser erradicada y permaneció hasta que, ya en épocas más recientes, fue redimensionada y puesta de nuevo en funcionamiento teórico y práctico. (Horrach, 2009). Estos grupos de personas organizadas formaban un Estado, ya que al participar en diversos temas se definieron los roles sociales y generaron agentes de cambio.

Esto implicaba a que los ciudadanos lucharan por sus derechos civiles, políticos y sociales abriendo la posibilidad de participar en la vida común, respetando los derechos de las demás personas y contribuyendo al bien común.

Después de todo, la ciudadanía moderna inicia en los años setenta del siglo XX en Europa y se renace en los años noventa del siglo XX como parte de la caída del socialismo conocido como la caída del muro de Berlín a finales de 1989, donde se da la apertura a un debate internacional para la creación de colectividades en un sentido de pertenencia a una comunidad y el otorgamiento de derechos políticos (Huntington, 1991).

A raíz de esto, Reyna (2006) plantea que los gobernantes de los estados comienzan a diseñar la forma de gobernar mediante un estado democrático, el gobierno se da cuenta que debe de delegar funciones en los ciudadanos dentro de las capacidades de los mismos para que estos tengan participación en las acciones que sean de su beneficio.

Pero también se deja claro que para ello se debe de distinguir entre una democracia participativa y una democracia representativa, la primera como una forma en la que los ciudadanos tienen una mayor participación en la toma de las decisiones políticas y la segunda es una forma de gobierno en la cual el pueblo tiene un poder político de forma indirecta, ejerciéndolo a través de unos representantes seleccionados mediante elecciones (Reyna, 2006).

En contraste con Canto (2010), la ciudadanía juega un papel importante en un estado democrático y al otorgarle derechos es que nace la figura de participación ciudadana. Lo explica también como las mediaciones entre sociedad y gobierno para que los distintos sujetos sociales intervengan con base en sus diversos intereses y valores influyendo así en 
las políticas públicas y en las estructuras de gobierno, siendo el reclamo del derecho a la intervención en los asuntos que les competen.

De ello nace el debate de diferentes autores de dar a conocer en qué consiste la participación ciudadana, por ello, Espinoza (2009) interpreta la participación ciudadana como la intervención de la sociedad civil en la esfera de lo público desde que integran una comunidad política.

Para Páres (2009) lo define como cualquier forma de acción colectiva, de reivindicación o de respuestas a las convocatorias formuladas desde el gobierno para incidir en las decisiones de política pública.

Por esta razón al estar enlazadas la democracia, ciudadanía y participación ciudadana los regímenes democráticos tienen que poner en práctica modelos que permitan una interacción entre estado y ciudadanía, esto generará que los servicios públicos sean de calidad, obteniendo un diálogo directo entre los representantes de gobierno y los actores participativos.

De igual manera, al proporcionar leyes que permiten la interacción entre ellos y los ciudadanos, surge la siguiente interrogante, ¿existe una verdadera percepción por parte de los ciudadanos de tener el conocimiento de la importancia de su participación en los asuntos de interés público y mediante qué forma pueden llevarlo a cabo?

Jones y Gaventa (2002) explican que en la teoría participativa existen elementos claros de democracia a través de la perspectiva de derechos, ya que la participación ciudadana deriva de una profundización democrática "derecho a tener derechos" y cada vez se encuentra más relacionada la participación con los gobiernos locales.

Por consiguiente, una participación ciudadana completa y efectiva es la que abre campo en el ámbito político a partir del control democrático del poder público.

De seguir así, se pueden obtener ciudadanos participantes que utilicen mecanismos formales de participación ciudadana como una forma de inclusión e intervención (individual o 
López C., $J$.

colectiva) en los procesos deliberativos y decisionales que conducen a las políticas y servicios públicos favorecería al mejoramiento de la actividad gubernamental.

\section{Gobierno local}

La participación ciudadana municipal surgió en respuesta a un sistema político centralizado, autoritario y deficiente donde se exigía la pluralidad cívica y política ya que en los municipios se encontraba el espacio para una democracia representativa y participativa, la eficiencia gubernamental y el desarrollo social. (Paz Cuevas, Pág. 130).

La CEPAL (2009) indica que la participación ciudadana en la gestión pública implica un proceso de construcción social de las políticas públicas. Siendo un derecho, responsabilidad y complemento de los mecanismos tradicionales de la representación.

Es por ello por lo que para mejorar la calidad de las políticas públicas es de gran importancia que la participación ciudadana sea temprana y oportuna, es decir, que esté presente desde el momento del diagnóstico de las problemáticas sociales para utilizar mecanismos que permitan la creación de políticas públicas que den respuestas a los conflictos sociales.

A la vez el beneficio de este tipo de implementación es que la gestión pública se democratiza, facilita el abordaje de conflictos y propicia los acuerdos, legitima y aumenta la efectividad de las decisiones políticas.

Para Paz Cuevas (s.f):

"El control que la ciudadanía ejercería sobre la Administración Pública municipal puede ser a través de las llamadas “contralorías sociales”, donde se vigila la aplicación y ejercicio de recursos, los avances en la elaboración de determinada obra pública, etcétera. Pueden existir otros órganos en los que la ciudadanía se disponga a recibir cuentas por parte de las autoridades, ya sea a través de los informes de labores o de ejercicio del gasto.” (Pág 130). 
Esto conllevaría al hecho de que la administración pública este regida por lo que determina la ley, a la vez, como está siendo observada por la población participativa, debe de estar bajo los criterios de calidad, eficacia y eficiencia en el uso de los recursos.

Por ejemplo, Díaz (2011) entabla que la participación ciudadana no es un fin, sino que es una herramienta que ayuda a cubrir déficits en la gestión por lo que el alcance o el nivel de personas involucradas no es un indicador de fiabilidad ya que dependen de la legitimidad de los mismos (pág 364); de esta manera, se valora la calidad de oferta participativa que hacen los gobiernos a la ciudadanía, de discurrir cuál o cuáles son las mejores formas de incorporar la participación y de dimensionar la medida en la que los ciudadanos realmente participan una vez que los gobiernos les brindan oportunidades para hacerlos.

Importancia de la participación ciudadana en los sistemas democráticos.

Una vez conceptualizado lo que se entiende por ciudadanía y la participación, se determina que es sustancial el involucramiento de los ciudadanos en las decisiones de los gobiernos.

Como lo explica Serrano (2015), la participación no se limita en el voto, sino que existen múltiples formas de tomar parte en los asuntos públicos, por lo que Villarreal (2010) hace mención a Cunill $(1991,1997)$ quien clasificó la participación en cuatro tipos, siendo estos: participación social, comunitaria, política y ciudadana.

Es decir, conceptualiza la participación ciudadana como:

"La participación ciudadana es aquella donde la sociedad posee una injerencia directa con el Estado; asimismo, tiene una visión más amplia de lo público. Esta participación está muy relacionada con el involucramiento de los ciudadanos en la administración pública. Los mecanismos de democracia directa (iniciativa de ley, referéndum, plebiscito y consultas ciudadanas), la revocación del mandato (recall) y la cooperación de los ciudadanos en la prestación de servicios o en la elaboración de políticas públicas, son formas de participación ciudadana” (Villarreal, 2010, pág 32). 
Por otra parte, todos los tipos de participación ciudadana son importantes dentro de la democracia ya que permiten vigilar y controlar la gestión de los gobernantes (Serrano, 2015), esto ayuda a que los indicadores de gobernabilidad sean positivos en los países que se respeta dicha participación.

De igual manera Serrano (2015) manifiesta que para que la participación ciudadana se consolide deben de existir las condiciones que permitan efectivizarla. Por ello, el sistema político deberá cumplir con cuatro requisitos que, según la autora, son de suma importancia para lograr el objetivo.

- El respeto de las garantías individuales.

- Los canales institucionales y marcos jurídicos.

- La información.

- La confianza por parte de los ciudadanos hacia las instituciones democráticas.

Otro ejemplo de ello es Aziz e Isunza (2017) donde establecen que la idea de participación ciudadana fue cimentando un lugar propio en el campo político a partir de la resemantización del control democrático del poder público. Esto implicaba el hecho de que el estado tendría que ser controlado por los representados, a través de mecanismos democráticos no electorales para mantener el ejercicio del gobierno representativo.

\section{Mecanismos formales de participación ciudadana}

Los mecanismos formales de participación ciudadana son las herramientas que permiten e incentivan la participación de las personas en las decisiones colectivas de la nación, así no sean parte de la administración pública o de un partido político. El objetivo es brindar garantías y beneficios para que el pueblo pueda incidir en cambios dentro del sistema judicial, ejecutivo y legislativo.

En primer lugar, los agentes participativos pueden resumir su decisión de participar o no participar a través de un complejo sistema de decisiones en torno a la reflexión de sus intereses. En tal sentido, los actores sin responsabilidades sociales y políticas tienden a 
considerar que el participar, aunque sea una vez en decisiones de carácter social, es más que suficiente.

Muchos de estos agentes participativos (ciudadanos) utilizan el sufragio como un medio por el cual pueden ejercer su derecho, pero esto va más allá, ya que no es únicamente el legitimar un sistema, sino que se debe de estar consciente del tipo de representante que se está eligiendo para la administración pública.

Siendo esta forma de participación una de las más utilizadas y conocidas, Bravo (2017) menciona que existen de forma amplia y diversa que existen mecanismos que los activados frecuentemente son: Los referéndums (plebiscitos y consultas populares) los utilizados por el $56 \%$ de los países y en segundo lugar las iniciativas ciudadanas directas, usados en $25 \%$ de los países.

Pues no se puede dejar de lado que el voto es uno de los mecanismos de participación democrática más conocido y utilizado, pero existen otros que permiten a la ciudadanía involucrarse en los asuntos públicos. Estos están integrados en una ley de participación ciudadana y su reglamento, la ley como garantía y el reglamento como la parte procedimental.

Aunque en la legislación de cada país se establecen cuáles son las herramientas con las que cuenta la población para que dentro de las facultades correspondientes puedan tener participación en los asuntos públicos Bravo (2017) indica que, la incorporación de mecanismos de participación ciudadana adicionales al sufragio adquiere relevancia.

El autor refiere que al identificar dos categorías de participación ciudadana institucionalizada se pretende que los ciudadanos influyan en los procesos de decisión con temas de interés público. Por lo que expresa:

"Se identifican dos categorías de participación ciudadana institucionalizada. La primera es la que realizan los ciudadanos para incidir en la conformación de los órganos de gobierno mediante la selección de sus representantes en votaciones, dar su apoyo a candidatos o partidos políticos, e incluso postularse por medio de candidaturas independientes. La segunda 
tiene que ver con la posibilidad de incidir en el diseño de políticas públicas y en la aprobación de leyes y reglamentos. Esto se realiza mediante instrumentos que permiten la intervención de la ciudadanía de manera directa, en vez de, por ejemplo, el trabajo de cabildeo que llevan a cabo organizaciones de la sociedad civil” (Bravo, 2017, pág 281).

Existen otros tipos de participación pero estas no están específicamente estipulada en la ley de participación ciudadana como un mecanismo, por ejemplo, en algunos países como parte de su constitución establecen la figura de la protesta, que es cuando los ciudadanos tienen el derecho fundamental de la reunión pacífica para exigir derechos o bien manifestar desacuerdo de alguna decisión, este tipo de mecanismo no se encuentra institucionalizada en la participación ciudadana pero es tomado en cuenta ya que se basa en favor o en contra de políticas públicas y disposiciones gubernamentales.

En general cada país establece en una ley que tipo de mecanismos le resulta más efectivo, en ámbito general se pueden mencionar los siguientes:

\section{CARACTERISTICAS DE LOS MECANISMOS FORMALES DE PARTICIPACIÓN.}

\begin{tabular}{|c|c|}
\hline Mecanismo & Tipos de resoluciones \\
\hline $\begin{array}{l}\text { Consulta } \\
\text { popular }\end{array}$ & $\begin{array}{l}\text { Plebiscito: La ciudadanía participa de las decisiones de carácter } \\
\text { administrativo } \\
\text { Referéndum: La ciudadanía participa en el sentido de la norma creada } \\
\text { o modificada en el ámbito legislativo. }\end{array}$ \\
\hline $\begin{array}{l}\text { Consulta } \\
\text { ciudadana }\end{array}$ & $\begin{array}{l}\text { Aprobación o rechazo de un acto o una decisión del interés colectivo de } \\
\text { la circunscripción estatal o municipal. } \\
\text { Debe contener la descripción específica del acto que se propone } \\
\text { consultar. } \\
\text { Exposición de motivos y razones por las cuales lo que se propone } \\
\text { someter a consulta ciudadana. }\end{array}$ \\
\hline $\begin{array}{l}\text { Iniciativa } \\
\text { popular }\end{array}$ & $\begin{array}{l}\text { Proyectos de creación, modificación, reforma, derogación o abrogación } \\
\text { de leyes que le corresponde decretar al Congreso del Estado o } \\
\text { reglamentos que sean competencia del poder ejecutivo o los } \\
\text { ayuntamientos. }\end{array}$ \\
\hline $\begin{array}{c}\text { Audiencia } \\
\text { pública }\end{array}$ & $\begin{array}{l}\text { Solicitan la adopción de determinados acuerdos o realización de ciertos } \\
\text { actos. } \\
\text { Recibir información sobre las actuaciones de los órganos de } \\
\text { administración pública y gobierno municipal. } \\
\text { Peticiones propuestas o quejas. }\end{array}$ \\
\hline
\end{tabular}




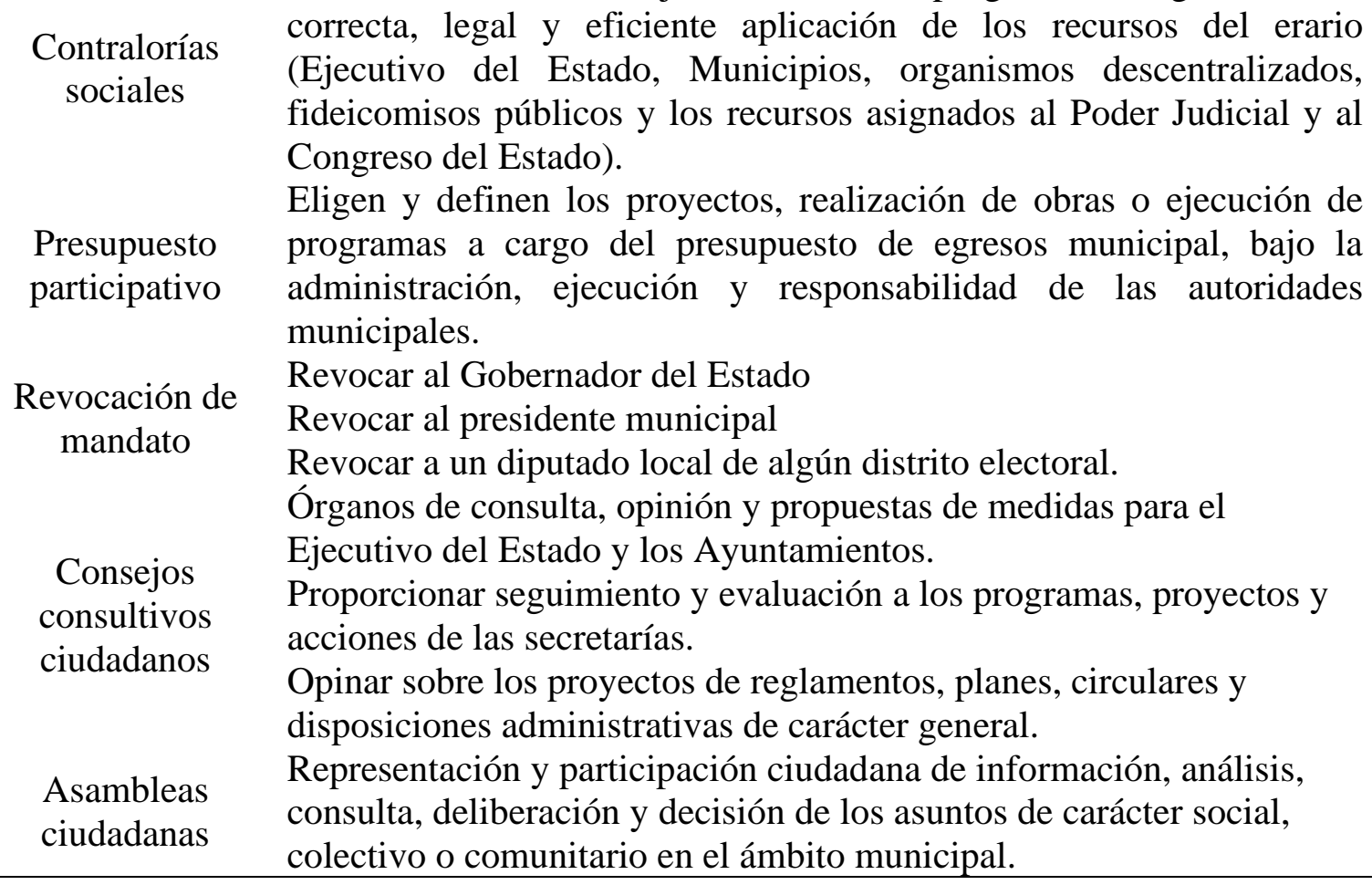

Fuente: Elaboración propia (2020) con base a Ley de Participación Ciudadana del Estado de

Nuevo León (Ley No. 107, Periódico Oficial \#62, 2016).

Esto viene de la mano con la democratización de un estado, por lo que uno de los retos para lograr la institucionalidad es la educación del ciudadano al crecer el régimen político requiere por consiguiente elevar el perfil de los ciudadanos, empleándose como parte de la calidad no tanto en conocimiento sino en civilidad.

John Stuart Mill (1977) remarca:

"Existen dos clases de ciudadanos los activos y los pasivos (...) los gobernantes prefieren a los ciudadanos pasivos porque es más fácil tener controlados a súbditos dóciles e indiferentes, pero la democracia necesita de los primeros” (pág 406).

Para lograrlo, es necesario una relación o vínculo entre sociedad y gobierno, de manera que la misma sociedad pueda creer en la gestión gubernamental. Uno de los medios son los espacios públicos, aquellos lugares donde la ciudadanía pueda ejercer sus derechos y como 
López C., $J$.

se menciona en apartados anteriores al involucrarse logra vigilar, controlar y aportar al sistema de gobierno.

Con esta apertura de espacios públicos es muy preciso para la promoción de las actividades en la que la ciudadanía puede opinar, Bustos (2004) expresa que el individuo genera su opinión acerca del gobierno, lo critica, cuestiona y propone, a esto se debe el reto, que el gobierno establezca los lugares donde el ciudadano pueda recurrir para lograr un beneficio de colectividad (pág 102).

Salinas de Gortari (2000) manifiesta que: "La confianza no es comprada; sino más bien prestada, puesto que puede perderse en cualquier momento y con increíble rapidez”.

A lo que Onofre (2004) interpreta que el ciudadano actúa por incentivos y que uno de ellos es la seguridad y compromiso del gobierno, eso aumentará la credibilidad de Estado de Derecho instaurado plenamente.

\section{Método.}

El presente artículo es de índole reflexivo por lo que no existe diseño, comprende una revisión de lo que han estipulado diversos autores sobre la participación ciudadana, los mecanismos de participación y los sistemas democráticos, es decir, se analiza desde un punto de referencia de los autores su teoría de participación

\section{CONCLUSIONES}

La creación de una ley sin sesgo en las formas de participación ciudadana provocará un sistema abierto cubriendo las necesidades de la ciudadanía. Una ley creada de manera sesgada es el producto de una disputa y conflicto de intereses políticos, esto sólo genera que los sistemas democráticos sean vulnerables, ya que los participantes perciben un problema en la respuesta gubernamental debido a que son leyes con vacíos en contenido o bien con un excesivo trámite burocrático para poder llevar a cabo alguna gestión, generando la improductividad en la administración pública. 
Abonado a esto, la falta de promoción, publicidad e implementación de estos mecanismos es la consecuencia del desconocimiento de una ley de participación ciudadana tanto en su forma normativa como reglamentaria, provocando que la ciudadanía no muestre interés con la instancia gubernamental en lo que corresponde a la agenda política.

Por lo que, para que se alcancen gobiernos más efectivos, eficientes y abiertos, los estados deben promover el ejercicio pleno de los ciudadanos en todos los ámbitos mediante mecanismos que permitan la interacción fluida entre estado y ciudadanía, con ello se lograría un sistema moderno en la gestión pública caracterizándose por la transparencia y promoción de la participación ciudadana.

\section{REFERENCIAS}

Arteta. (2008). El saber del ciudadano. Las nociones capitales de democracia.

Aziz Nassif, A., \& Isunza Vera, E. (2017). Las razones del fracaso democrático. Ciudad de Mexico: Colección México.

Bautista, J. (2015). Gobernabilidad, ciudadanía y democracia participativa.

Bourgon, J. (2010). Propósito público, autoridad gubernamental y poder colectivo.

Bravo Escobar, E. (2017). Mecanismos de participación ciudadana en perspectiva comparada. UNAM

Brugué, Q. (2004). Modernizar la administración desde la izquierda: burocracia, nueva gestión pública y administración deliberativa.

Bueno, M. (s.f.). Aristóteles y el Ciudadano. 2018. México, México.

Cabrero. (2005). Acción pública y desarrollo local.

Camou. (2009). Participación ciudadana, gestión social y gobernabilidad: Gobernando el conflicto a nivel local.

Chac, C. (2010). Participación ciudadana en las políticas públicas.

Diaz Aldret, A. (2017). Participación ciudadana en la gestión y en las políticas públicas. Scielo.

Espinoza, M. (2009). La participación ciudadana como una relación socio-estatal acotada por la concepción de democracia y ciudadanía. Andamios, 71-109.

Gortari, H., \& Hernández, R. (1988). Memoria y encuentros: la ciudad de México y el Distrito Federal. Una historia compartida.

Horrach, J. (2009). Sobre el concepto de ciudadanía: historia y modelos.

Huntington, S. (1996). El orden político en las sociedades en cambio.

ILPES/CEPAL, L. W. (2011). Políticas públicas: formas de evaluación. La antigua, Guatemala.

Jones, E., \& Gaventa, J. (2002). Concepts of Citizenship.

Ley No. 107, Peródico Oficial \#62. (13 de mayo de 2016). Ley de Participación Ciudadana del Estado de Nuevo León. Nuevo León, Monterrey, México. 
Onofre, J. I. (2004). Confianza y Estado de derecho. Principales incentivos para la participación ciudadana institucional. 1.

Paz Cueva, C. (s,f). La participación ciudadana municipal en México: Factor para el desarrollo y la eficiencia gubernamental. Revistas UNAM, 130.

Reyna. (2006). El destino d ela participación ciudadana en México. Revista de Posgrado en Derecho de la UNAM.

Serrano, A. (2015). La participación ciudadana en México.

Villarreal, M. (2010). Participación ciudadana y políticas píublicas. 